\title{
Electronic properties of iron-boron pairs in crystalline silicon by temperature- and injection-level-dependent lifetime measurements
}

\author{
Jens E. Birkholz and Karsten Bothe \\ Institut für Solarenergieforschung Hameln/Emmerthal (ISFH), Am Ohrberg 1, D-31860 Emmerthal, \\ Germany \\ Daniel Macdonald \\ Department of Engineering, The Australian National University, Canberra, ACT 0200, Australia \\ Jan Schmidt ${ }^{\mathrm{a})}$ \\ Institut für Solarenergieforschung Hameln/Emmerthal (ISFH), Am Ohrberg 1, D-31860 Emmerthal, \\ Germany
}

(Received 7 December 2004; accepted 1 March 2005; published online 3 May 2005)

\begin{abstract}
Iron-boron pairs in crystalline silicon are studied by measuring the recombination lifetime as a function of injection density, doping concentration, and temperature. The characteristic crossover point of the injection-level-dependent carrier lifetime curves measured before and after optical dissociation of the iron-boron pairs is analyzed to determine the energy level as well as the electronand hole-capture cross sections of the acceptor level of iron-boron pairs, assuming known recombination parameters for interstitial iron. The doping concentration dependence of the crossover point gives an electron-capture cross section of $(1.4 \pm 0.2) \times 10^{-14} \mathrm{~cm}^{2}$, while the temperature dependence results in a hole-capture cross section in the range from 0.5 $\times 10^{-15}$ to $2.5 \times 10^{-15} \mathrm{~cm}^{2}$ and an energy level of $(0.26 \pm 0.02) \mathrm{eV}$ below the conduction-band edge. () 2005 American Institute of Physics. [DOI: 10.1063/1.1897489]
\end{abstract}

\section{INTRODUCTION}

The recombination properties of iron and iron-boron pairs play an important role in silicon device technology. ${ }^{1}$ FeB pairs occur naturally in iron-contaminated, boron-doped $p$-type silicon wafers as an equilibrium state. These pairs can be dissociated by energy supply (e.g., illumination, current injection, or heating) into positively charged interstitial iron $\left(\mathrm{Fe}_{i}^{+}\right)$and negatively charged substitutional boron $\left(\mathrm{B}_{s}^{-}\right)$. After ceasing the energy supply, the equilibrium state is reached due to an association process, driven by Coulombic attraction between the mobile interstitial iron and the immobile substitutional boron ion. ${ }^{1,2}$ As recently reviewed by Istratov et $a .^{3}{ }^{3}$ deep-level transient spectroscopy (DLTS) shows that there are at least two different energy levels related to FeB pairs, one shallow donor level at $E_{V}+0.1 \mathrm{eV}$ and one acceptor level between $E_{C}-0.23$ and $E_{C}-0.29 \mathrm{eV}$. The latter state is believed to be the dominant recombination center, as it is deeper.

In order to characterize any defect and its impact on the bulk lifetime, the electronic defect parameters, i.e., the energy level and the capture cross sections, must be known. While the electronic properties of isolated interstitial iron $\left(\mathrm{Fe}_{i}\right)$ are well established, ${ }^{2}$ the scatter in the experimentally determined recombination parameters of the acceptor state of $\mathrm{FeB}$ pairs is very large. ${ }^{4,5}$ Injection-level-dependent lifetime spectroscopy has been applied recently in several defect characterization studies, because of its high sensitivity to electronically active recombination centers in the silicon band gap. ${ }^{4,6,7}$ Applying this measurement technique to

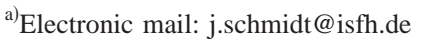

boron-doped iron-contaminated silicon wafers results in very different injection-level-dependent lifetime curves for the dissociated and the associated FeB state, leading to a welldefined characteristic crossover point. ${ }^{8}$

The simultaneous determination of the three FeB parameters, i.e., the electron- and hole-capture cross sections $\sigma_{n}(\mathrm{FeB})$ and $\sigma_{p}(\mathrm{FeB})$ and the energy level $E(\mathrm{FeB})$, by fitting the injection-level-dependent experimental curves using Shockley-Read-Hall (SRH) theory ${ }^{9,10}$ is ambiguous and suffers from large uncertainties, particularly because additional knowledge about the total iron concentration is necessary.

Therefore, in this paper we apply an approach, which is independent of the total iron concentration, to determine the acceptor energy level and capture cross sections of FeB pairs by analyzing the position of the crossover point as a function of doping level and temperature. Our method only utilizes the accurately known electronic parameters of interstitial iron, which can be regarded as an internal calibration standard.

\section{THEORY}

Comparing the injection-level-dependent lifetime curves for the dissociated $\left(\mathrm{Fe}_{i}\right)$ and associated $(\mathrm{FeB})$ state, one observes a very different injection-level dependence for these two states (in the following denoted by "as" and "dis") leading to a characteristic crossover point. At this point the carrier lifetimes of both states are equal:

$$
\tau_{\text {as }}=\tau_{\text {dis }} .
$$

Since both $\mathrm{Fe}_{i}$ and $\mathrm{FeB}$ are very effective recombination centers, we consider only SRH recombination and neglect all 
other recombination processes (e.g., Auger recombination, radiative recombination). The pairing reaction of iron-boron pairs

$$
\left(\mathrm{Fe}_{i}\right)^{+}+\left(\mathrm{B}_{s}\right)^{-} \rightleftarrows\left(\mathrm{Fe}_{i} \mathrm{~B}_{s}\right)^{0}
$$

can be regarded as a chemical equilibrium reaction. Thus, in reality we neither deal with a pure dissociated nor with a pure associated state, but mixed states with a certain fraction of the total iron content being in the form of interstitial iron $\left[N\left(\mathrm{Fe}_{i}\right)\right]$ and a certain fraction being bound to boron $[N(\mathrm{FeB})]$. form

Therefore, we can write Eq. (1) more precisely in the

$$
\frac{1}{\tau_{\mathrm{SRH}, \mathrm{as}}(\mathrm{FeB})}+\frac{1}{\tau_{\mathrm{SRH}, \mathrm{as}}\left(\mathrm{Fe}_{i}\right)}=\frac{1}{\tau_{\mathrm{SRH}, \mathrm{dis}}(\mathrm{FeB})}+\frac{1}{\tau_{\mathrm{SRH}, \mathrm{dis}}\left(\mathrm{Fe}_{i}\right)} .
$$

Using SRH statistics for Eq. (3) (Refs. 9 and 10) gives a third-order polynomial in the injection level $\Delta n$, with only one physical solution for the crossover point $\Delta n_{\mathrm{COP}}$. The detailed calculations are given in Appendix A, where we also show that for a $p$-type boron-doped silicon wafer with doping concentration $p_{0} \geqslant 10^{14} \mathrm{~cm}^{-3}$ the following simplified expression holds:

$$
\begin{aligned}
\Delta n_{\mathrm{COP}}= & \underbrace{\frac{\sigma_{n}^{-1}(\mathrm{FeB})-\sigma_{n}^{-1}\left(\mathrm{Fe}_{i}\right)}{\sigma_{p}^{-1}\left(\mathrm{Fe}_{i}\right)} p_{0}}_{\Delta n_{\mathrm{DD}}} \\
& +\underbrace{\frac{\sigma_{p}\left(\mathrm{Fe}_{i}\right)}{\sigma_{p}(\mathrm{FeB})} N_{C} \exp \left(-\frac{E_{C}-E(\mathrm{FeB})}{k T}\right)}_{\Delta n_{\mathrm{TD}}} .
\end{aligned}
$$

In the derivation of Eq. (4) we have used that $\sigma_{p}\left(\mathrm{Fe}_{i}\right)$ is much smaller than all other capture cross sections involved. ${ }^{3}$ Furthermore, a possible temperature dependence of the capture cross sections is not taken into account by our model. According to Eq. (4), $\Delta n_{\mathrm{COP}}$ can be split up into a dopingdependent part $\Delta n_{\mathrm{DD}}$ [left-hand term in Eq. (4)] and a temperature-dependent part $\Delta n_{\mathrm{TD}}$ [right-hand term in Eq. (4)]: $\Delta n_{\mathrm{COP}}=\Delta n_{\mathrm{DD}}+\Delta n_{\mathrm{TD}}$.

Hence, by measuring the crossover point $\Delta n_{\mathrm{COP}}$ for samples of different doping densities $p_{0}$ at a constant temperature, we are able to determine the prefactor $\left[\sigma_{n}^{-1}(\mathrm{FeB})\right.$ $\left.-\sigma_{n}^{-1}\left(\mathrm{Fe}_{i}\right)\right] / \sigma_{p}^{-1}\left(\mathrm{Fe}_{i}\right)$ in the doping-dependent part in Eq. (4). Furthermore, temperature-dependent measurements of one sample (constant doping concentration) should give the energy level $E(\mathrm{FeB})$ and the value of $\sigma_{p}\left(\mathrm{Fe}_{i}\right) / \sigma_{p}(\mathrm{FeB})$.

Assuming known values for the capture cross sections of $\mathrm{Fe}_{i}$, temperature- and doping-dependent measurements of the crossover point enable us to determine all recombination parameters of the $\mathrm{FeB}$ pairs.

\section{EXPERIMENT}

\section{A. Sample preparation}

We use boron-doped $p$-type float-zone (FZ) silicon wafers of different resistivities $(0.26,0.6,1$, and $25 \Omega \mathrm{cm})$ in our experiments. After initial etching and cleaning to remove any surface damage and contamination, ${ }^{56} \mathrm{Fe}$ is implanted using a low-energy implanter $(\sim 70 \mathrm{keV})$. In order to obtain a uniform iron distribution throughout the bulk, the samples are annealed at $900{ }^{\circ} \mathrm{C}$ for $1 \mathrm{~h}$. The implantation doses are $10^{12} \mathrm{~cm}^{-2}$ for the $25-\Omega \mathrm{cm}$ sample and $10^{11} \mathrm{~cm}^{-2}$ for all other samples. For a 300- $\mu$ m-thick wafer these doses correspond to a total iron concentration of $3 \times 10^{13}$ and 3 $\times 10^{12} \mathrm{~cm}^{-3}$, respectively. Both values are below the solubility limit of $\mathrm{Fe}_{i}$ in $\mathrm{Si}$ at $900{ }^{\circ} \mathrm{C}\left(\sim 5 \times 10^{13} \mathrm{~cm}^{-3}\right){ }^{3}$ Hence, the applied doses are not expected to create significant iron precipitates. Precipitation could especially occur at the $\mathrm{Si} / \mathrm{SiO}_{2}$ interface during annealing. This is avoided by removing the native oxide prior to the annealing and adding nitrogen gas during the furnace process to avoid oxidation. By rapid cooling in air, the iron is frozen in its interstitial state. Although the implantation energy is quite low, to avoid implantation damage in the silicon lattice, several microns of silicon are etched off the wafer surface after the annealing to remove the implanted region and to make sure that the lifetime measurements are not distorted due to nonuniform iron distributions caused by gettering sites.

Finally, the samples are passivated by plasma-enhanced chemical-vapor deposition (PECVD) of silicon nitride $\left(390{ }^{\circ} \mathrm{C}\right.$ for $\left.10 \mathrm{~min}\right)$. Since precipitation problems are avoided at this relatively low temperature and short deposition time, this method has a clear advantage over other passivation techniques such as oxidation, which operate at higher temperatures. Furthermore, PECVD SiN films provide a very low surface recombination velocity and are also stable under light exposure and temperature treatment below $250{ }^{\circ} \mathrm{C}$. Therefore, these $\mathrm{SiN}$ films are most suitable for bulk lifetime measurements. ${ }^{11}$

\section{B. Measurement technique}

We apply the contactless quasi-steady-state photoconductance (QSSPC) technique ${ }^{12}$ to measure the injectionlevel-dependent effective carrier lifetimes of our ironcontaminated samples. Carrier generation is caused by the light of a photo-flash light source (Quantum QFlash X2). The flash decays exponentially with a time constant of about $2.1 \mathrm{~ms}$, which is over two orders of magnitude greater than the measured lifetimes. Hence, quasi-steady-state conditions prevail.

The sample conductance changes due to excess carrier generation and is detected by a coil of an rf bridge circuit via inductive coupling. This circuit generates an output voltage signal proportional to the excess carrier density $\Delta n$. The time-dependent intensity of the light pulse is determined by measuring the short-circuit current of a calibrated reference cell in order to determine the generation rate $G$. The effective lifetime is then calculated by $\tau=\Delta n / G$, since $G$ equals the recombination rate $U$ under steady-state conditions.

At carrier concentrations $\leqslant 10^{13} \mathrm{~cm}^{-3}$ we observe significant trapping effects. These trapping sites may occur in the bulk or at the $\mathrm{Si} / \mathrm{SiN}$ interface and trap electrons from the conduction band without directly contributing to recombination. ${ }^{13}$ It will be shown in the following that our 


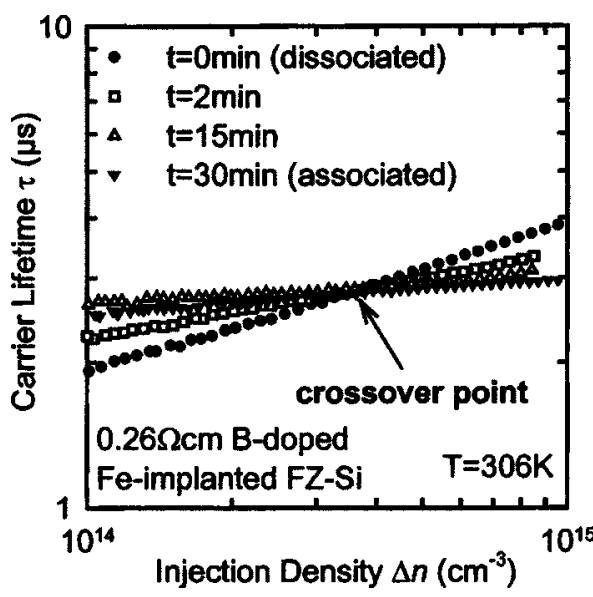

FIG. 1. Injection-level-dependent carrier lifetime curves of a $0.26-\Omega \mathrm{cm}$ iron-implanted boron-doped silicon wafer measured with the QSSPC method at a constant temperature of $306 \mathrm{~K}$. The curves show different injection-level dependencies for different times after light-soaking, leading to a characteristic crossover point.

range of interest is well-above injection densities of $10^{13} \mathrm{~cm}^{-3}$. Hence, trapping does not affect our lifetime data.

The original QSSPC setup (Sinton Consulting, Inc) has been complemented with a temperature-controlled stage in order to enable temperature-dependent lifetime measurements. ${ }^{14}$ The sample is positioned onto a Pyrex ${ }^{\mathrm{TM}}$ glass disk, which is located about $1 \mathrm{~mm}$ away from the top of the photoconductance-sensor coil by means of insulating spacers. The sample can be heated via contact to a brass ring, which is heated using a resistive element. The heating power is adjusted by a proportional controller and the sample temperature is measured by a thermocouple mounted on its surface. A slight temperature increase of the coil, which could occur in spite of good thermal isolation of the sample stage, does not affect the calibration of the rf bridge circuit and, hence, does not hamper the measurement. ${ }^{14}$

In order to determine the crossover point, we dissociate the FeB pairs by illuminating the iron-implanted silicon samples for about 2 min with light from a halogen lamp (intensity $\sim 0.5 \mathrm{~W} / \mathrm{cm}^{2}$ ). The sample is placed on a cooled aluminum block $\left(\sim 10{ }^{\circ} \mathrm{C}\right)$ to avoid a significant temperature increase, since this would result in a faster association after switching off the light source. ${ }^{2}$ After light-soaking, we measure the injection-level-dependent recombination lifetime $\tau(\Delta n)$ using the QSSPC method. ${ }^{12}$ We repeat the measurements a couple of times with a break of several minutes between each measurement until the associated state is reached. The sample temperature is kept constant during the entire experiment.

\section{RESULTS AND DISCUSSION}

In Fig. 1, the lifetime data for the $0.26-\Omega \mathrm{cm}$ sample is plotted versus the injection density. One observes a significant increase in lifetime with increasing injection level in the dissociated state $(t=0 \mathrm{~min})$ and a reduced slope for the subsequent measurements, corresponding to succeeding states of FeB pairing $(t=2,15$, and $30 \mathrm{~min}$ after illumination). From this set of $\tau(\Delta n)$ curves the position $\Delta n_{\mathrm{COP}}=(3.5 \pm 0.2)$

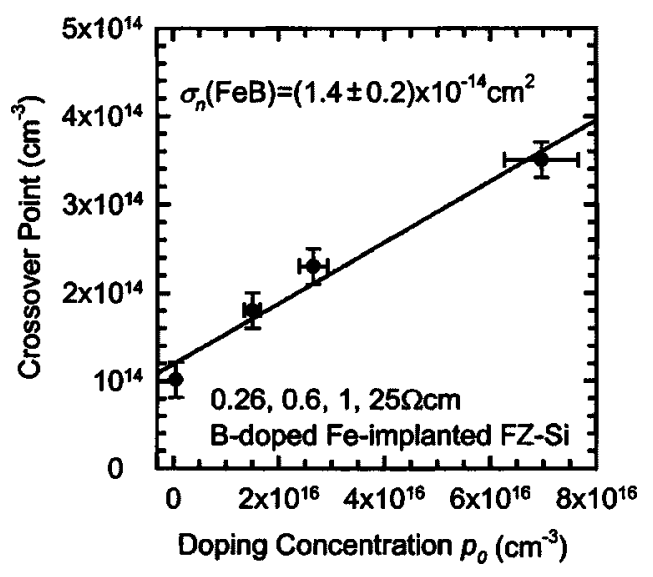

FIG. 2. Crossover point for samples of different doping concentrations. According to theory, the crossover point depends linearly on the doping concentration. The slope of the linear fit gives the prefactor of the dopingdependent part $\Delta n_{\mathrm{DD}}$ [see Eq. (4)], and therefore the electron capture cross section $\sigma_{n}(\mathrm{FeB})$ of iron-boron pairs.

$\times 10^{14} \mathrm{~cm}^{-3}$ of the crossover point can be determined accurately. The same kind of measurement is performed for all samples $(0.26,0.6,1$, and $25 \Omega \mathrm{cm})$.

As shown in Fig. 2, plotting the crossover point over the doping concentration shows a linear dependence as expected from the theory. According to Eq. (4), the slope of the linear fit (obtained using the Lewenberg-Marquardt algorithm) gives the prefactor of $p_{0}$, and the fit offset yields the temperature-dependent part. Thus, using the reported values of $\sigma_{n}\left(\mathrm{Fe}_{i}\right)$ and $\sigma_{p}\left(\mathrm{Fe}_{i}\right),{ }^{3}$ we are able to determine the electron-capture cross section of the FeB pair. The fit shown in Fig. 2 results in an accurate value for $\sigma_{n}$ of $(1.4 \pm 0.2)$ $\times 10^{-14} \mathrm{~cm}^{2}$. However, the value of $\sigma_{p}(\mathrm{FeB})$ still depends on the energy level $E(\mathrm{FeB})$. Figure 3 shows the injection-leveldependent lifetime data for the $1-\Omega \mathrm{cm}$ sample for two different temperatures ( 304 and $352 \mathrm{~K}$ ). The shift of the crossover point to higher injection densities with increasing temperature can clearly be observed. A separation of energy level and hole-capture cross section is possible by temperature-dependent measurements of $\Delta n_{\mathrm{COP}}$, according to Eq. (4). We consider the logarithm of $\Delta n_{\mathrm{COP}}-\Delta n_{\mathrm{DD}}$ :

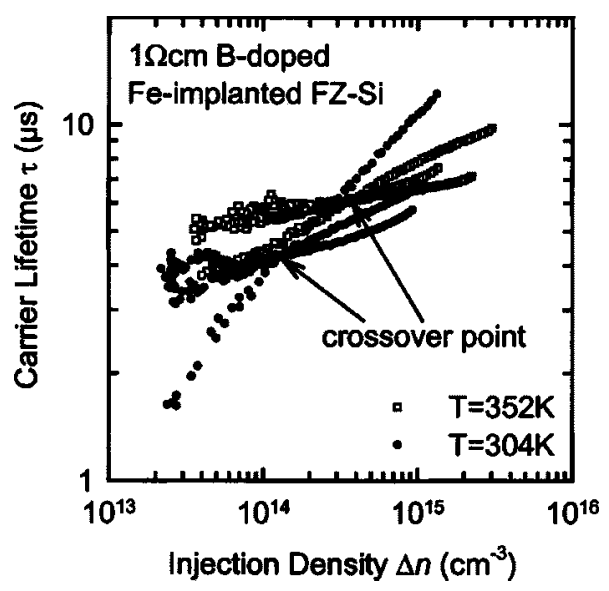

FIG. 3. Injection-level-dependent lifetime data (QSSPC) of a $1-\Omega \mathrm{cm}$ ironimplanted boron-doped silicon wafer for two different temperatures (304 and $352 \mathrm{~K}$ ). The shift of the crossover point to higher injection densities with increasing temperature can clearly be observed. 


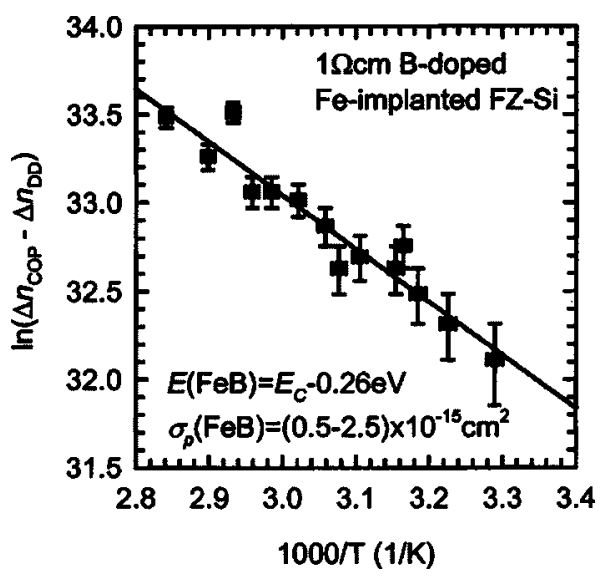

FIG. 4. Temperature-dependent measurement of the crossover point for a $1-\Omega \mathrm{cm}$ iron-implanted boron-doped silicon wafer. The Arrhenius plot of the temperature-dependent part $\Delta n_{\mathrm{TD}}=\Delta n_{\mathrm{COP}}-\Delta n_{\mathrm{DD}}$ [see Eq. (5)] can be fitted linearly. The slope of the linear fit yields the energy level $E(\mathrm{FeB})$ of ironboron pairs, and the hole-capture cross section $\sigma_{p}(\mathrm{FeB})$ is determined from the offset.

$$
\begin{aligned}
\ln (\Delta & \left.n_{\mathrm{COP}}-\frac{\sigma_{n}^{-1}(\mathrm{FeB})-\sigma_{n}^{-1}\left(\mathrm{Fe}_{i}\right)}{\sigma_{p}^{-1}\left(\mathrm{Fe}_{i}\right)} p_{0}\right) \\
=- & \frac{E_{C}-E(\mathrm{FeB})}{k T}+\ln \left(\frac{\sigma_{p}\left(\mathrm{Fe}_{i}\right) N_{C}}{\sigma_{p}(\mathrm{FeB})}\right) .
\end{aligned}
$$

As an example, the temperature-dependent experimental data for the $1-\Omega \mathrm{cm}$ sample $\left([\mathrm{Fe}]=3.7 \times 10^{12} \mathrm{~cm}^{-3}\right)$ is plotted in the form of Eq. (5) (Arrhenius plot) in Fig. 4, and a linear fit gives $\sigma_{p}(\mathrm{FeB})$. Measurement errors could occur due to temperature variations (estimated to be smaller than $1 \mathrm{~K}$ in the current experimental setup) and inaccuracies in the determination of the crossover point (estimated to be smaller than $\left.0.2 \times 10^{14} \mathrm{~cm}^{-3}\right)$.

According to Eq. (5), the energy level $E(\mathrm{FeB})$ is given by the slope of the linear fit function, whereas the holecapture cross section $\sigma_{p}(\mathrm{FeB})$ can be calculated from the offset, if $\sigma_{p}\left(\mathrm{Fe}_{i}\right)$ is known. Using the reported value for $\sigma_{p}\left(\mathrm{Fe}_{i}\right)$ of $7 \times 10^{-17} \mathrm{~cm}^{2}$, we obtain $E(\mathrm{FeB})=E_{C}$ $-(0.26 \pm 0.02) \mathrm{eV}$ and $\sigma_{p}(\mathrm{FeB}) \approx 1.1 \times 10^{-15} \mathrm{~cm}^{2}$. The uncertainty range for $\sigma_{p}(\mathrm{FeB})$ is less than one order of magnitude $\left(0.5 \times 10^{-15}-2.5 \times 10^{-15} \mathrm{~cm}^{2}\right)$. These values agree quite well with values reported in the literature. ${ }^{3,4}$ In this experi- ment, we do not account for a temperature dependence of $\sigma_{p}(\mathrm{FeB})$ and $\sigma_{p}\left(\mathrm{Fe}_{i}\right)$, although the temperature dependence of $\sigma_{p}\left(\mathrm{Fe}_{i}\right)$ is known. ${ }^{3}$ However, assuming similar temperature dependencies for $\sigma_{p}(\mathrm{FeB})$ and $\sigma_{p}\left(\mathrm{Fe}_{i}\right)$, this dependence is compensated in Eq. (5), and taking the reported value for $\sigma_{p}\left(\mathrm{Fe}_{i}\right)$ at room temperature yields the corresponding value for $\sigma_{p}(\mathrm{FeB})$.

With the method described above, the value of $E(\mathrm{FeB})$ can be determined with an error of only $10 \%$. The inaccuracy in the $\sigma_{n}(\mathrm{FeB})$ determination is about $20 \%$, whereas the value for $\sigma_{p}(\mathrm{FeB})$ can only be given with an accuracy of approximately one order of magnitude, which is comparable to the uncertainty obtained in DLTS experiments. Without making any assumptions on the values for $\sigma_{p}\left(\mathrm{Fe}_{i}\right)$ and $\sigma_{n}\left(\mathrm{Fe}_{i}\right)$, our method still allows for a determination of $E(\mathrm{FeB})$ and the hole-capture cross section ratio $\sigma_{p}\left(\mathrm{Fe}_{i}\right) / \sigma_{p}(\mathrm{FeB})=0.07$ with an uncertainty range of $0.03-$ 0.14 , as well as for a determination of $\sigma_{p}\left(\mathrm{Fe}_{i}\right)\left[1 / \sigma_{n}(\mathrm{FeB})\right.$ $\left.-1 / \sigma_{n}\left(\mathrm{Fe}_{i}\right)\right]=(3.5 \pm 0.4) \times 10^{-3}$. Table I shows a comparison of our results (COP stands for the crossover point measurement method) with other reported values for $E(\mathrm{FeB})$, $\sigma_{n}(\mathrm{FeB})$, and $\sigma_{p}(\mathrm{FeB})$. For $\mathrm{Fe}_{i}$ data see Ref. 3. While the energy level $E(\mathrm{FeB})$ agrees very well with the results obtained by other measurement techniques such as DLTS or electron paramagnetic resonance (EPR), this is not the case for the capture cross sections. However, our values of $\sigma_{n}(\mathrm{FeB})$ and $\sigma_{p}(\mathrm{FeB})$ coincide very well with the results obtained by injection-level-dependent lifetime spectroscopy (IDLS) measurements. ${ }^{4}$ The uncertainty range for $\sigma_{n}(\mathrm{FeB})$ and $\sigma_{p}(\mathrm{FeB})$ in Ref. 4 is given by $(3 \pm 2) \times 10^{-14}$ and $(2 \pm 1) \times 10^{-15} \mathrm{~cm}^{2}$, respectively.

A clear evidence for the accuracy of our method is that we are able to fit all injection-level-dependent lifetime data by applying our results for $E(\mathrm{FeB}), \sigma_{n}(\mathrm{FeB})$, and $\sigma_{p}(\mathrm{FeB})$ to SRH statistics. This is shown in Fig. 5 for a $1-\Omega \mathrm{cm}$ ironimplanted boron-doped silicon wafer (at $304 \mathrm{~K}$ ). The simultaneous fitting of all three curves is possible with parameters $E(\mathrm{FeB})=E_{C}-0.26 \mathrm{eV}, \quad \sigma_{n}(\mathrm{FeB})=1.4 \times 10^{-14} \mathrm{~cm}^{2}, \quad$ and $\sigma_{p}(\mathrm{FeB})=1.4 \times 10^{-15} \mathrm{~cm}^{2}$. A comparison to the values in Table I shows that there is only a small modification of $\sigma_{p}(\mathrm{FeB})$, well within the uncertainty range, necessary to fit the data. The total iron concentration was set at $N_{T}=2.8$ $\times 10^{12} \mathrm{~cm}^{-3}$ for the fit, which deviates less than $15 \%$ from

TABLE I. Energy levels and capture cross sections for the acceptor state of FeB pairs in silicon. The values obtained by our crossover point method (COP) are compared with reported values obtained by other measurement techniques such as deep-level transient spectroscopy (DLTS), injection-level-dependent lifetime spectroscopy (IDLS), electron paramagnetic resonance (EPR), surface photo voltage (SPV), and the Elymat technique. Values for interstitial iron $\left[E\left(\mathrm{Fe}_{i}\right)=E_{V}+0.38 \mathrm{eV}, \sigma_{p}\left(\mathrm{Fe}_{i}\right)=7 \times 10^{-17} \mathrm{~cm}^{2}, \sigma_{n}\left(\mathrm{Fe}_{i}\right)=4 \times 10^{-14} \mathrm{~cm}^{2}\right]$ have been taken from Ref. 3.

\begin{tabular}{lccccc}
\hline \hline $\begin{array}{c}\text { Measurement } \\
\text { technique }\end{array}$ & $\begin{array}{c}\text { Energy level } \\
(\mathrm{eV})\end{array}$ & $\begin{array}{c}\sigma_{n} \\
\left(\mathrm{~cm}^{2}\right)\end{array}$ & $\begin{array}{c}\sigma_{p} \\
\left(\mathrm{~cm}^{2}\right)\end{array}$ & $\sigma_{n} / \sigma_{p}$ & Reference \\
\hline COP & $E_{C}-0.26$ & $1.4 \times 10^{-14}$ & $1.1 \times 10^{-15}$ & 13 & This work \\
IDLS & $E_{C}-0.23$ & $3 \times 10^{-14}$ & $2 \times 10^{-15}$ & 15 & Ref. 4 \\
SPV, ELYMAT & $E_{C}-0.29$ & $2.5 \times 10^{-15}$ & $3 \times 10^{-14}$ & 0.1 & Ref. 5 \\
DLTS (T=90 K) & $E_{C}-0.23$ & $1.8 \times 10^{-15}$ & $5.4 \times 10^{-15}$ & 0.3 & Ref. 15 \\
DLTS & $E_{C}-0.29$ & $\cdots$ & $\cdots$ & $\cdots$ & Ref. 16 \\
EPR & $E_{C}-0.275$ & $\cdots$ & $\cdots$ & $\cdots$ & Ref. 17 \\
\hline \hline
\end{tabular}




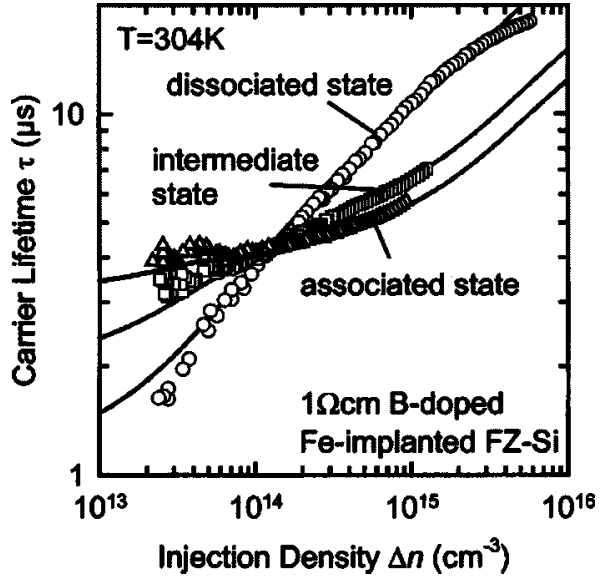

FIG. 5. Injection-level-dependent lifetime data (QSSPC) of an ironimplanted boron-doped silicon wafer $(1 \Omega \mathrm{cm})$. The parameters $E(\mathrm{FeB})$, $\sigma_{n}(\mathrm{FeB})$, and $\sigma_{p}(\mathrm{FeB})$ obtained in this work are applied to fit the data with SRH statistics.

the estimated value of $2.5 \times 10^{12} \mathrm{~cm}^{-3}$ (implantation dose: $10^{11} \mathrm{~cm}^{-2}$, wafer thickness: $400 \mu \mathrm{m}$ ), which is well within the concentration uncertainty range of the ion implantation technique. This shows that our method is also applicable to determine the iron concentration in boron-doped silicon. The free variables in the analysis are the ratios of the concentration of interstitial iron and iron-boron pairs $N_{T}\left(\mathrm{Fe}_{i}\right) /$ $N_{T}(\mathrm{FeB})$, which were determined to be 1.55 (dissociated state), 0.33 (intermediate state), and 0.08 (associated state). Further injection- and time-dependent measurements could also give information about the association kinetics of $\mathrm{FeB}$ pairs.

\section{CONCLUSION}

We have presented a method for determining the recombination parameters (energy level and electron- and holecapture cross sections) of iron-boron pairs in silicon by analyzing the crossover point obtained from injection-leveldependent lifetime measurements before and after optical dissociation. Our approach represents a good alternative to DLTS in terms of determining the energy level. Compared to the IDLS approach, i.e., fitting the injection-level-dependent lifetime data using SRH theory, the advantage of our method is its internal calibration based only on the well-known parameters for $\mathrm{Fe}_{i}$. A more sophisticated model should also take a possible temperature dependence of the capture cross sections into account. However, a clear benefit of our method is that it is not restricted just to FeB pairs, but can be used whenever a defect occurs in two states for which the injection-level-dependent lifetime curves show a crossover point. This is believed to be a quite common behavior of iron-acceptor pairs. Schmidt and MacDonald ${ }^{18}$ have recently observed a crossover point for iron-gallium $(\mathrm{FeGa})$ and ironindium (FeIn) pairs, respectively. Since boron and gallium are the most frequently used dopants in $p$-type silicon and iron is one of the most important impurities in silicon, our method is applicable to many relevant situations.

\section{ACKNOWLEDGMENTS}

Funding was provided by the State of Lower Saxony and the German Ministry for the Environment, Nature Conservation and Nuclear Safety (BMU). D. M. acknowledges the financial support of the Australian Research Council and J. S., the support of the Alexander von Humboldt Foundation. The ISFH is a member of the German Forschungsverbund Sonnenenergie.

\section{APPENDIX A: MATHEMATICAL ANALYSIS}

Assuming negligible trapping of carriers, and hence $\Delta n$ $=\Delta p$, the carrier lifetime related to a single-level defect in the band gap is, according to SRH theory, given by ${ }^{19}$

$\tau_{X}(\Delta n)=\frac{\tau_{n_{0}}(X)\left(p_{0}+p_{1}(X)+\Delta n\right)+\tau_{p_{0}}(X)\left(n_{0}+n_{1}(X)+\Delta n\right)}{p_{0}+n_{0}+\Delta n}$,

where $X$ denotes the type of defect, i.e., $\mathrm{Fe}_{i}$ or $\mathrm{FeB}$. We use the same notation as Blakemore, ${ }^{19}$ i.e.,

$$
\begin{aligned}
& p_{1}(X)=N_{V} \exp \left(-\frac{E(X)-E_{V}}{k T}\right), \\
& n_{1}(X)=N_{C} \exp \left(-\frac{E_{C}-E(X)}{k T}\right), \\
& \tau_{n_{0}}(X)=\left(v_{\mathrm{th}} N(X) \sigma_{n}(X)\right)^{-1}, \quad \tau_{p_{0}}(X)=\left(v_{\mathrm{th}} N(X) \sigma_{p}(X)\right)^{-1} .
\end{aligned}
$$

The values for the thermal velocity $v_{\text {th }}$ and the effective densities of states in the conduction band $N_{C}$ and in the valence band $N_{V}$ are taken from Ref. 20.

$N(X), E(X), \sigma_{n}(X)$, and $\sigma_{p}(X)$ are the defect density, the energy level, and the capture cross sections for electrons and holes, respectively. In the following, we neglect all other recombination processes (e.g., Auger recombination, radiative recombination).

First, let us assume that all iron atoms are bound to boron in the associated state and that a complete dissociation of these $\mathrm{FeB}$ pairs into $\mathrm{Fe}_{i}$ and $\mathrm{B}_{s}$ occurs. Combining Eqs. (1) and (A1) gives

$$
\begin{array}{r}
\tau_{n_{0}}(\mathrm{FeB})\left(p_{0}+p_{1}(\mathrm{FeB})+\Delta n\right)+\tau_{p_{0}}(\mathrm{FeB})\left(n_{0}+n_{1}(\mathrm{FeB})+\Delta n\right) \\
\quad=\tau_{n_{0}}\left(\mathrm{Fe}_{i}\right)\left(p_{0}+p_{1}\left(\mathrm{Fe}_{i}\right)+\Delta n\right)+\tau_{p_{0}}\left(\mathrm{Fe}_{i}\right)\left(n_{0}+n_{1}\left(\mathrm{Fe}_{i}\right)+\Delta n\right) .
\end{array}
$$

Solving Eq. (A3) for $\Delta n$ gives the crossover point (COP):

$$
\Delta n_{\mathrm{COP}}=\frac{\tau_{n_{0}}\left(\mathrm{Fe}_{i}\right)\left(p_{0}+p_{1}\left(\mathrm{Fe}_{i}\right)\right)+\tau_{p_{0}}\left(\mathrm{Fe}_{i}\right)\left(n_{0}+n_{1}\left(\mathrm{Fe}_{i}\right)\right)-\tau_{n_{0}}(\mathrm{FeB})\left(p_{0}+p_{1}(\mathrm{FeB})\right)-\tau_{p_{0}}(\mathrm{FeB})\left(n_{0}+n_{1}(\mathrm{FeB})\right)}{\tau_{n_{0}}(\mathrm{FeB})+\tau_{p_{0}}(\mathrm{FeB})-\tau_{n_{0}}\left(\mathrm{Fe}_{i}\right)-\tau_{p_{0}}\left(\mathrm{Fe}_{i}\right)} .
$$


This expression can be further simplified (see discussion below). As mentioned in the text, there are mixed states in reality, but the total iron content $N(\mathrm{Fe})=N\left(\mathrm{Fe}_{i}\right)+N(\mathrm{FeB})$ always remains constant. Thus, we have

$$
\frac{1}{\tau_{\mathrm{SRH}, \mathrm{as}}(\mathrm{FeB})}+\frac{1}{\tau_{\mathrm{SRH}, \mathrm{as}}\left(\mathrm{Fe}_{i}\right)}=\frac{1}{\tau_{\mathrm{SRH}, \mathrm{dis}}(\mathrm{FeB})}+\frac{1}{\tau_{\mathrm{SRH}, \mathrm{dis}}\left(\mathrm{Fe}_{i}\right)} \text {. }
$$

The left-hand side of Eq. (A5) corresponds to the state before, the right-hand side to the state after (incomplete) dissociation. Using Eq. (A1), we obtain after some rearrangements

$$
\begin{aligned}
(\Delta n)^{3} & +\left[\frac{w(\mathrm{FeB}) q\left(\mathrm{Fe}_{i}\right)+w\left(\mathrm{Fe}_{i}\right) q(\mathrm{FeB})}{w(\mathrm{FeB}) w\left(\mathrm{Fe}_{i}\right)}+\frac{q\left(\mathrm{Fe}_{i}\right)-q(\mathrm{FeB})}{w\left(\mathrm{Fe}_{i}\right)-w(\mathrm{FeB})}\right] \\
& \times(\Delta n)^{2}+\left[\frac{q\left(\mathrm{Fe}_{i}\right) q(\mathrm{FeB})}{w\left(\mathrm{Fe}_{i}\right) w(\mathrm{FeB})}\right. \\
& \left.+\frac{\left(q\left(\mathrm{Fe}_{i}\right)-q(\mathrm{FeB})\right)\left(w(\mathrm{FeB}) q\left(\mathrm{Fe}_{i}\right)+w\left(\mathrm{Fe}_{i}\right) q(\mathrm{FeB})\right)}{\left(w\left(\mathrm{Fe}_{i}\right)-w(\mathrm{FeB})\right) w(\mathrm{FeB}) w\left(\mathrm{Fe}_{i}\right)}\right] \\
& \times \Delta n+\left[\frac{q\left(\mathrm{Fe}_{i}\right) q(\mathrm{FeB})\left(q\left(\mathrm{Fe}_{i}\right)-q(\mathrm{FeB})\right)}{w\left(\mathrm{Fe}_{i}\right) w(\mathrm{FeB})\left(w\left(\mathrm{Fe}_{i}\right)-w(\mathrm{FeB})\right)}\right]=0,
\end{aligned}
$$

with

$$
\begin{aligned}
& w(X):=\frac{1}{\sigma_{n}(X)}+\frac{1}{\sigma_{p}(X)} \\
& q(X):=\frac{p_{0}+p_{1}(X)}{\sigma_{n}(X)}+\frac{n_{0}+n_{1}(X)}{\sigma_{p}(X)} .
\end{aligned}
$$

This is a third-order polynomial, but instead of using Cardano's formula for a general solution we check whether our result from above [Eq. (A4)] solves Eq. (A6). This is indeed the case, so the general expression for the crossover point becomes

$$
\Delta n_{\mathrm{COP}}=\frac{q\left(\mathrm{Fe}_{i}\right)-q(\mathrm{FeB})}{w(\mathrm{FeB})-w\left(\mathrm{Fe}_{i}\right)} .
$$

Obviously, the crossover point depends only on the temperature, the doping density, the energy levels, and the capture cross sections of $\mathrm{Fe}_{i}$ and $\mathrm{FeB}$ and not on the iron concentration $N(\mathrm{Fe})$. Equation (A6) has two other solutions, but they are negative, and hence not of physical interest.

We consider a $p$-type boron-doped silicon wafer with doping concentration $p_{0} \geqslant 10^{14} \mathrm{~cm}^{-3} . n_{1}\left(\mathrm{Fe}_{i}\right) \approx 10^{7} \mathrm{~cm}^{-3}$, $p_{1}\left(\mathrm{Fe}_{i}\right) \approx 10^{13} \mathrm{~cm}^{-3}, \quad p_{1}(\mathrm{FeB}) \approx 10^{4} \mathrm{~cm}^{-3}, \quad$ and $n_{0} \leqslant 10^{6}$ $\mathrm{cm}^{-3}$ can be neglected in Eq. (A4). Thus, we obtain the simplified equation

$$
\begin{aligned}
\Delta n_{\mathrm{COP}}= & \frac{\sigma_{n}^{-1}(\mathrm{FeB})-\sigma_{n}^{-1}\left(\mathrm{Fe}_{i}\right)}{\sigma_{p}^{-1}\left(\mathrm{Fe}_{i}\right)} p_{0}+\frac{\sigma_{p}\left(\mathrm{Fe}_{i}\right)}{\sigma_{p}(\mathrm{FeB})} N_{C} \\
& \times \exp \left(-\frac{E_{C}-E(\mathrm{FeB})}{k T}\right) .
\end{aligned}
$$

Additionally, we have used here that $\sigma_{p}\left(\mathrm{Fe}_{i}\right)$ is much smaller than all the other capture cross sections involved. ${ }^{3}$

${ }^{1}$ K. Graff and H. Pieper, J. Electrochem. Soc. 128, 669 (1981).

${ }^{2}$ L. C. Kimerling and J. L. Benton, Physica B \& C 116, 297 (1983).

${ }^{3}$ A. A. Istratov, H. Hieslmair, and E. R. Weber, Appl. Phys. A: Mater. Sci. Process. 69, 13 (1999).

${ }^{4}$ D. Macdonald, A. Cuevas, and J. Wong-Leung, J. Appl. Phys. 89, 7932 (2001).

${ }^{5}$ D. Walz, J.-P. Joly, and G. Kamarinos, Appl. Phys. A: Mater. Sci. Process. 62, 435 (1996).

${ }^{6}$ J. Schmidt and A. Cuevas, J. Appl. Phys. 86, 3175 (1999).

${ }^{7}$ S. Rein, T. Rehrl, W. Warta, and S. W. Glunz, J. Appl. Phys. 91, 2059 (2001).

${ }^{8}$ D. H. Macdonald, L. J. Geerlings, and A. Azzizi, J. Appl. Phys. 95, 1021 (2004).

${ }^{9}$ W. Shockley and W. T. Read, Jr., Phys. Rev. 87, 835 (1952).

${ }^{10}$ R. N. Hall, Phys. Rev. 87, 387 (1952).

${ }^{11}$ J. Schmidt and M. Kerr, Sol. Energy Mater. Sol. Cells 65, 585 (2001).

${ }^{12}$ R. A. Sinton and A. Cuevas, Appl. Phys. Lett. 69, 2510 (1996).

${ }^{13}$ D. Macdonald and A. Cuevas, Appl. Phys. Lett. 74, 1710 (1999).

${ }^{14}$ J. Schmidt, Appl. Phys. Lett. 82, 2178 (2003).

${ }^{15}$ H. Lemke, Phys. Status Solidi A 64, 215 (1981).

${ }^{16}$ S. D. Brotherton, P. Bradley, and A. Gill, J. Appl. Phys. 57, 1941 (1985).

${ }^{17}$ S. Ghatnekar-Nilsson, M. Kleverman, P. Emanuelsson, and H. G. Grimmeiss, Mater. Sci. Forum 143-147, 171 (1994).

${ }^{18} \mathrm{~J}$. Schmidt and D. Macdonald, (unpublished).

${ }^{19}$ J. S. Blakemore, Semiconductor Statistics (Dover, New York, 1962).

${ }^{20}$ M. A. Green, Solar Cells (The University of New South Wales, Kensington, 1992). 